agree that dose reduction is a reasonable option and one that may be associated with continued clinical benefit.

Alice T. Shaw, M.D., Ph.D.

Massachusetts General Hospital Cancer Center

Boston, MA

ashawl@partners.org
Benjamin J. Solomon, M.B., B.S., Ph.D.

Peter MacCallum Cancer Centre

Melbourne, VIC, Australia

Since publication of their article, the authors report no further potential conflict of interest.

DOI: 10.1056/NEJMc1415359

\title{
Early versus On-Demand Tube Feeding in Pancreatitis
}

TO THE EDITOR: Bakker et al. (Nov. 20 issue) ${ }^{1}$ found no benefit of early enteral nutrition in patients with predicted severe acute pancreatitis. Clinical trials involving patients with this condition are hampered by the low positive predictive value of current prognostic scoring systems, resulting in the inclusion of many patients who ultimately have mild acute pancreatitis and do not require early enteral nutrition. ${ }^{2} \mathrm{~A}$ composite end point allowed for sample-size reduction but ultimately resulted in an underpowered study, owing to the inequality between the individual end points. ${ }^{3}$ Death and infection have vastly different clinical significance, given that persistent organ failure, not infection, is the primary cause of death in patients with severe acute pancreatitis. ${ }^{4,5}$ The timing, type, and volume of fluid administered were not detailed in this study. Individual centers across this consortium may have different practices with regard to fluid resuscitation that potentially biased the results toward the null hypothesis. Enteral nutrition has consistently been shown to have a benefit in patients with severe acute pancreatitis, but we are still no closer to optimizing patient selection and the timing of enteral nutrition.

Robert A. Moran, M.D,

Ruben Hernaez, M.D., Ph.D.

Vikesh K. Singh, M.D.

Johns Hopkins Medical Institutions

Baltimore, MD

vsingh1@jhmi.edu

No potential conflict of interest relevant to this letter was reported.

1. Bakker OJ, van Brunschot S, van Santvoort HC, et al. Early versus on-demand nasoenteric tube feeding in acute pancreatitis. N Engl J Med 2014;371:1983-93.

2. Mounzer R, Langmead CJ, Wu BU, et al. Comparison of existing clinical scoring systems to predict persistent organ failure in patients with acute pancreatitis. Gastroenterology 2012;142: 1476-82.

3. Cordoba G, Schwartz L, Woloshin S, Bae H, Gфtzsche PC. Definition, reporting, and interpretation of composite outcomes in clinical trials: systematic review. BMJ 2010;341:c3920.
4. Guo Q, Li A, Xia Q, et al. The role of organ failure and infection in necrotizing pancreatitis: a prospective study. Ann Surg 2014;259:1201-7.

5. Besselink MG, van Santvoort HC, Boermeester MA, et al. Timing and impact of infections in acute pancreatitis. Br J Surg 2009;96:267-73.

DOI: 10.1056/NEJMc1415356

TO THE EDITOR: The study by Bakker et al. requires careful interpretation. First, the actual delivery site of enteral nutrition is unclear. The authors did not disclose in the article that the original ethics committee approval was for tube feeding into the stomach, not the jejunum. Furthermore, feeding tubes were dislodged in $40 \%$ of the patients, which many would consider to be a rather high rate for leading research centers. Second, only one third of the study patients had actual, as opposed to predicted, severe or critical acute pancreatitis (i.e., persistent organ failure, infected pancreatic necrosis, or both). ${ }^{1}$ Hence, two thirds of the patients in the study were not posed to benefit from tube feeding. ${ }^{2}$ Third, the lack of superiority of feeding within 24 hours after presentation versus feeding at 72 hours (or later) after presentation does not mean that the latter should be deemed the preferred nutritional strategy. The weight of evidence indicates that the most effective time to start feeding in patients with acute pancreatitis is between 24 and 72 hours after presentation, and the exact timing of feeding in an individual patient is influenced by the adequacy of intravenous fluid resuscitation and opiate use. ${ }^{3-5}$

Maxim S. Petrov, M.D., Ph.D.
John A. Windsor, M.B., Ch.B., M.D.
University of Auckland
Auckland, New Zealand
max.petrov@gmail.com
No potential conflict of interest relevant to this letter was re-
ported.
1. Dellinger EP, Forsmark CE, Layer P, et al. Determinant-based
classification of acute pancreatitis severity: an international
multidisciplinary consultation. Ann Surg 2012;256:875-80.


2. Petrov MS. Gastric feeding and "gut rousing" in acute pancreatitis. Nutr Clin Pract 2014;29:287-90.

3. Petrov MS, Pylypchuk RD, Uchugina AF. A systematic review on the timing of artificial nutrition in acute pancreatitis. $\mathrm{Br} J$ Nutr 2009;101:787-93.

4. Sun JK, Li WQ, Ke L, et al. Early enteral nutrition prevents intra-abdominal hypertension and reduces the severity of severe acute pancreatitis compared with delayed enteral nutrition: a prospective pilot study. World J Surg 2013;37:2053-60.

5. Petrov MS, Windsor JA. Nutritional management of acute pancreatitis: the concept of 'gut rousing.' Curr Opin Clin Nutr Metab Care 2013;16:557-63.

DOI: 10.1056/NEJMc1415356

THE AUTHORS REPLY: The limited accuracy of prognostic scoring systems in acute pancreatitis is addressed in the letters by Moran et al. and by Petrov and Windsor. However, currently there is no better tool available during triage, when clinicians need to make decisions regarding any type of early intervention. This limitation does not undermine the validity of the study results. The sample size was estimated on the basis of studies that used similar predictive scoring systems in similar patient populations with similar clinical outcomes. ${ }^{1}$ Furthermore, this approach reflects how clinical decision making for patients with acute pancreatitis is done in everyday practice and therefore, in our opinion, is relevant to practicing clinicians.

Moran et al. question the composition of the primary end point. Our study was designed to show a reduction in the rate of infection because infection has a major effect on the outcome of patients with acute pancreatitis. ${ }^{2}$ No significant differences were found in the individual components of the primary end point, which strongly suggests that the proposed inequality does not hamper the overall power of the study.

In the complex setting of a large, multicenter trial, subtle differences in treatment among centers may occur, including with regard to fluid resuscitation. However, a randomized study design and stratification according to study center balance out such differences between treatment groups.
In response to Petrov and Windsor: the first version of the protocol indeed included nasogastric instead of nasojejunal feeding. After critical appraisal of the available evidence at the time, we decided to switch to nasojejunal feeding to minimize the risk of aspiration. This decision was made before the start of patient recruitment and hence did not influence outcome (for details, see www.isrctn.com/ISRCTN18170985). The rate of tube dislocation is similar to rates found in the literature. ${ }^{3,4}$

Our study did not show that starting an oral diet 72 hours after presentation is the most effective strategy for all patients with acute pancreatitis. However, our results show that routine early tube feeding in all patients at high risk for severe pancreatitis does not improve outcome and that the implementation of on-demand tube feeding will reduce patient discomfort and costs.

OlafJ. Bakker, M.D.

University Medical Center Utrecht

Utrecht, the Netherlands

Marc G. Besselink, M.D., Ph.D.

Amsterdam Medical Center

Amsterdam, the Netherlands

Hein G. Gooszen, M.D., Ph.D.

Radboud University Medical Center

Nijmegen, the Netherlands

Since publication of their article, the authors report no further potential conflict of interest.

1. Bakker OJ, van Santvoort HC, van Brunschot S, et al. Pancreatitis, very early compared with normal start of enteral feeding (PYTHON trial): design and rationale of a randomised controlled multicenter trial. Trials 2011;12:73.

2. Wu BU, Johannes RS, Kurtz S, Banks PA. The impact of hospital-acquired infection on outcome in acute pancreatitis. Gastroenterology 2008;135:816-20.

3. Seder CW, Stockdale W, Hale L, Janczyk RJ. Nasal bridling decreases feeding tube dislodgment and may increase caloric intake in the surgical intensive care unit: a randomized, controlled trial. Crit Care Med 2010;38:797-801.

4. Gerritsen A, Besselink MG, Cieslak KP, et al. Efficacy and complications of nasojejunal, jejunostomy and parenteral feeding after pancreaticoduodenectomy. J Gastrointest Surg 2012;16: 1144-51.

DOI: 10.1056/NEJMc1415356

\section{High-Cost Generic Drugs — Implications for Patients and Policymakers}

TO THE EDITOR: The Perspective article by Alpern et al. (Nov. 13 issue) ${ }^{1}$ states that manufacturers of new generic drugs can have delays before the Office of Generic Drugs of the Food and Drug Administration (FDA) approves their products.
Over the years, the increasing number of applications submitted to the FDA for the review of generic drugs resulted in a backlog that drove the establishment of the Generic Drug User Fee Amendments (GDUFA) of 2012.2 The GDUFA 